\title{
Construction of Continuous and Discrete Distribution Laws for Risk Assessment in Information Systems
}

\author{
Yu.Yu. Gromov*, Yu.V. Minin, O.G.Ivanova and H.L. Majeed \\ Tambov State Technical University, Institute of Automation and Information Technology, Tambov, Russia,
}

Received 10 May 2019; Accepted 24 April 2020

\begin{abstract}
The paper constructs continuous and discrete distribution laws, used to assess risks in information systems. Generalized expressions for continuous distribution laws with maximum entropy are obtained. It is shown that in the general case the entropy depends also on the type of moments used to determine the numerical characteristics of the distribution law. Also, probabilistic models have been developed to analyze the sequence of independent trials with three outcomes. Expressions for their basic numerical characteristics are obtained, as well as for calculating the probabilities of occurrence of the corresponding events.
\end{abstract}

Keywords: information system, distribution, risk, random variable

\section{Introduction}

At the present stage of the development of society, which is characterized by the intensive introduction of information systems in virtually all areas of activity, issues related to the assessment of the risks that occur during their operation are of particular importance. When analyzing and assessing risks, issues related to the definition of distribution laws are of the greatest importance. The given work is devoted to questions of construction of distribution laws.

In the modeling of information systems, risk is a random variable and is described by a probability distribution on a given set [1-3]. In contrast to experiments conducted in physics, where there is a possibility of their multiple conduct, the conditions of the functioning of information systems are characterized by a constant impact of negative external influences and are constantly changing [4], and consequently the repetition of the experiment under the same conditions is practically impracticable. The laws of probability distribution of risk events, as a rule, do not correspond to the law of the normal Gaussian distribution [5$6]$.

\section{Construction of continuous distribution laws with the maximum entropy}

Entropy coefficient is often used [7-8] with the classification of distribution laws of random continuous value (RV) with number characteristics.

$$
\delta_{e}=\frac{1}{2 \sigma} \exp (H)
$$

*E-mail address: gromovtambov@yandex.ru ISSN: $1791-2377$ @ 2020 School of Science, HU. All rights reserved. doi:10.25103/jestr.133.01
In the formula (1) $\sigma=\sqrt{\mu_{2}}$ is standard deviation, and $\mu_{2}$ is the second central power moment for this distribution law; value $\mathrm{H}$ - entropy, which is defined by the definition:

$H=-\int_{-\infty}^{\infty} p(x) \ln (p(x)) d x$

$p(x)$ - density of probability distribution (PDD) SV. Entropy coefficient has the maximum value for Gaussian law $\left(\delta_{e}=2.066\right.$ ), for uniform law $-\delta_{e}=1.73$, for Koshi distribution $-\delta_{e}=0$ etc.

The entropy value does not depend on shift parameter, to simple computation let's consider, that it is equal to zero. Firstly we need to find distribution law from unilateral laws of distribution of unlimited $\mathrm{RV}$, for which entropy value $H(2)$ reaches the maximum with the following limitations, imposed on probability density $p(x)$ :

$$
p(x) \geq 0, \int_{0}^{\infty} p(x) d x=1, \int_{0}^{\infty} x^{v} p(x) d x=\beta^{v} / v,
$$

where $\beta$ - scale parameter; $v$ - value of maximum existing primary direct moment. Here and next we'll consider positive power moment as a direct moment in accordance with (3), and negative power moment as a reverse moment.

To find the extremum we'll use the method of indefinite Lagrange multipliers [9]. We need to maximize:

$$
\int_{0}^{\infty}\left[-p(x) \ln (p(x))+\lambda_{1} p(x)+\lambda_{2} x^{v} p(x)\right] d x
$$

inserting Lagrange multipliers $\lambda_{1}$ and $\lambda_{2}$, considering the limitations (3) and must be defined. Equating the result of 
variation integrand expression in (4) when $p(x)=0$, we'll take the equation relatively to $p(x)$ :

$$
-\ln (p(x))-1+\lambda_{1}+\lambda_{2} x^{v}=0
$$

So, the density $p(x)$, which is satisfies (3) and maximizes $H$, can be found from the equation (5)

$$
p(x)=\exp \left(\lambda_{1}-1+\lambda_{2} x^{v}\right)
$$

Substituting (6) in (3) instead of $p(x)$, we'll take from integrating:

$$
\begin{aligned}
& \exp \left(\lambda_{1}-1\right) \frac{\Gamma(1 / v)}{v\left(-\lambda_{2}\right)^{1 / v}}=1, \\
& \exp \left(\lambda_{1}-1\right) \frac{\Gamma(1 / v)}{v\left(-\lambda_{2}\right)^{1+1 / v}}=\beta^{v} / v .
\end{aligned}
$$

$$
\begin{aligned}
& \text { From } \quad \text { we } \quad \text { find, } \\
& \lambda_{2}=-1 / \beta^{v}, \exp \left(\lambda_{1}-1\right)=v /(\beta \Gamma(1 / v)) \text {. Consequently } \\
& p(x)=\frac{v}{\beta \Gamma(1 / v)} \exp \left(-\frac{x^{v}}{\beta^{v}}\right), \quad 0<x<\infty,
\end{aligned}
$$

where $\tilde{\mathrm{A}}(z)$ - gamma function.

From (8) follows, that if exists only the first beginning direct moment $(v=1)$, than exponential law has the maximum entropy; if there are two moments $(v=2)$, then unilateral Gaussian law; and if all direct moments exist $(v \rightarrow \infty)$, than unilateral uniform law. Indeed, the limiting moment (8) with $(v \rightarrow \infty)$ is a unilateral uniform law $p(x)=\beta^{-1}, 0<x<\beta$. So, if all direct moments exist, then uniform law has the maximum entropy from unilateral distribution laws of RV.

Analogically for bilateral symmetry laws of distribution unlimited RV can be shown, that if the first $v$ of absolute central direct moments, then the probability density has the maximum entropy:

$$
p(x)=\frac{0.5 v}{\beta \Gamma(1 / v)} \exp \left(-\frac{|x|^{v}}{\beta^{v}}\right), \quad-\infty<x<\infty .
$$

From (9) follows, that if only first absolute central moment exists $(v=1)$, then Laplace distribution has the biggest entropy; if there are two moments $(v=2)$, then Gaussian law; and if all direct moments exist $(v \rightarrow \infty)$, then uniform law. Indeed, the limiting case for (9) is a uniform law $p(x)=0,5 \beta^{-1}, \quad-\beta<x<\beta$. So, if all direct moments exist, then uniform law has the biggest entropy from bilateral symmetry distribution laws of RV. Considered private cases of bilateral laws with the maximum entropy coincide with already known laws (Laplace and Gaussian), which have maximum entropy, that confirms the correctness of received results.
From analysis of received expressions (8) and (9) follows, that for increasing the amount of information about evaluating parameters of distribution laws with big length (with long "tails") with the help of a method of moments is necessary to use direct moments of lesser order, including fractional order. If the parameters of distribution laws lesser length are used, then it is necessary to use direct moment of higher order.

Let's find from unilateral distribution laws of unlimited RV such distribution law, with which entropy value $H$ reaches maximum with the following limitations, imposed on probability density $p(x)$ :

$$
\begin{aligned}
& p(0)=0, p(x) \geq 0, \\
& \int_{0}^{\infty} p(x) d x=1, \int_{0}^{\infty} x^{-v} p(x) d x=\beta^{v} / v,
\end{aligned}
$$

where $v$ - value of maximum existing beginning reverse moment. Considering this an entropy is defined by an expression:

$$
\begin{aligned}
& H=-\int_{0}^{\infty} y^{-2} p(1 / y) \ln \left(y^{-2} p(1 / y)\right) d y= \\
& =-\int_{0}^{\infty} p(x) \ln \left(x^{2} p(x)\right) d x
\end{aligned}
$$

where $y^{-2} p(1 / y)$ - a probability density RV $\eta$, which is reverse to $\xi$, which has the probability density $p(x)$. As a result of using the method of indefinite Lagrange numerators we'll receive following expression for distribution law with the maximum entropy

$$
\begin{array}{r}
p(x)=\frac{v \exp (x)}{\beta \Gamma(1 / v)} \exp \left(-\frac{\exp (v x)}{\beta^{v}}\right), \\
-\infty<x<\infty .
\end{array}
$$

The limiting case for (12) with $v \rightarrow \infty$ (all reverse moments exist) is a unilateral distribution law of limitations down from RV $p(x)=1 / \beta x^{2}, 1 / \beta<x<\infty$.

Let's define from bilateral distribution laws of RV such distribution law, for which entropy value $H$ reaches the maximum with the following limitations, imposed on probability density $p(x)$

$$
\begin{aligned}
& p(x) \geq 0, \quad \int_{-\infty}^{\infty} p(x) d x=1, \\
& \int_{-\infty}^{\infty} \exp (v x) p(x) d x=\beta^{v} / v,
\end{aligned}
$$

where $v-$ a value of maximum existing primary direct exponential moment. Considering this an entropy $\mathrm{H}$ is defined by the expression

$$
H=-\int_{-\infty}^{\infty} p(x) \ln (\exp (-x) p(x)) d x
$$


As a result of using the method of indefinite Lagrange numerators we'll receive the following expression for distribution law with the maximum entropy.

$$
p(x)=\frac{v \exp (x)}{\beta \Gamma(1 / v)} \exp \left(-\frac{\exp (v x)}{\beta^{v}}\right),-\infty<x<\infty .
$$

The limiting case for (15) when $v \rightarrow \infty$ (all direct exponential moments exist) is a distribution law of bordered above RV $p(x)=\exp (x) / \beta,-\infty<x<\ln (\beta)$.

Now let's find from bilateral distribution laws of unlimited RV such distribution law, for which the value of entropy $H$ reaches maximum with the following limitations, imposed on probability density $p(x)$ :

$$
\begin{aligned}
& p(x) \geq 0, \quad \int_{-\infty}^{\infty} p(x) d x=1, \\
& \int_{-\infty}^{\infty} \exp (-v x) p(x) d x=\beta^{v} / v,
\end{aligned}
$$

where $v-$ a value of maximum existing primary reverse exponential moment. Considering this an entropy $H$ is defined by the expression

$$
H=-\int_{-\infty}^{\infty} p(x) \ln (\exp (x) p(x)) d x .
$$

As a result of using the method of indefinite Lagrange numerators we'll receive the following expression for distribution law with the maximum entropy.

$$
p(x)=\frac{v \exp (-x)}{\beta \Gamma(1 / v)} \exp \left(-\frac{\exp (-v x)}{\beta^{v}}\right),-\infty<x<\infty .
$$

The limiting case for (18) when $v \rightarrow \infty$ (all direct exponential moments exist) is a distribution law of bordered above RV $p(x)=\exp (-x) / \beta,-\ln (\beta)<x<\infty$

From the analysis of expressions (15) and (18) follows, that exponential transformation of RV leads to transformation of form parameter $v$ in scale parameter, and $\beta$ parameter in shift parameter.

Finally let's define from unilateral distribution laws of unlimited RV such distribution law, for which the value of entropy $H$ reaches maximum with the following limitations, imposed on probability density $p(x)$ :

$$
\begin{aligned}
& p(0)=0, \quad p(x) \geq 0, \\
& \int_{0}^{\infty} p(x) d x=1, \int_{0}^{\infty}|\ln (x)|^{v} p(x) d x=\beta^{v} / v,
\end{aligned}
$$

where $v-$ a value of maximum existing primary direct logarithmic moment. Considering this an entropy $H$ is defined by the expression

$$
H=-\int_{0}^{\infty} p(x) \ln (x \cdot p(x)) d x
$$

As a result of using the method of indefinite Lagrange numerators we'll receive the following expression for distribution law with the maximum entropy.

$$
p(x)=\frac{v}{2 \beta \Gamma(1 / v) x} \exp \left(-\frac{|\ln (x)|^{v}}{\beta^{v}}\right), 0<x<\infty .
$$

From (21) it follows, that if only two absolute logarithmic moments exist ( $v=2)$, then logarithmic normal law has the biggest entropy. If $v \rightarrow \infty$ (all absolute primary moments exist), then (21) is transforming in Shannon law for limitations from above and down of RV $p(x)=0,5 / \beta x$, $\exp (-\beta)<x<\exp (\beta)$. It is necessary to notice, that with logarithmic transformation of $\mathrm{RV}$ scale parameter $\beta$ transforms in form parameter and shift parameter transforms in scale parameter.

In general case, if RV $\eta$ connected with RV $\xi$ by a ratio $y=f(x)$ and known $\mathrm{PDD} p(y)$ of continuous $\mathrm{RV} \xi$, then PDD $p(x)$ can be found by a method of functional transformation with the help of expression

$$
p(x)=p(y) \cdot\left|\frac{d y}{d x}\right| .
$$

At this an entropy $H$, considering (22) and ratio

$$
H=-\int_{\Theta} p(y) \ln (p(y)) d y
$$

will be defined by an expression

$$
H=-\int_{\Omega} p(x) \ln (q(x) \cdot p(x)) d x,
$$

where $q(x)=|d y / d x|^{-1} ; \Theta$ and $\Omega$ - areas of existence RV $\eta$ and $\xi$ respectively.

\section{Distributions arising in the analysis of the sequence of independent tests with three outputs}

Next, consider the development of a probabilistic model of a sequence of independent trials with three outcomes, which becomes particularly important in the formation of estimates of the information security of information processing systems [10].

Most often during the test, it is taken into account that its result is either event $\mathrm{A}$ or the opposite event $\mathrm{C}$. The probability of event $\mathrm{A}$ in any test is independent of the outcomes of all other tests (the tests are independent) and equal to the probability (this is ensured by the same set of conditions for each test). This scheme of tests was first considered by J. Bernoulli and bears his name [11-14].

The probability $P_{A}(k)$ of the fact, that event $A$ in $N$ tests will come precisely $k$ times $(k=1,2, \ldots, N)$ is defined by Bernoulli's formula [13-15]

$$
P_{A}(k)=\frac{N !}{(N-k) ! k !} p^{k}(1-p)^{N-k},
$$


which represents binomial distribution. In $N=1$ it transforms in Bernoulli's distribution.

$$
P_{A}(k)=p^{k}(1-p)^{1-k}
$$

The limiting case of binomial distribution, when $p \rightarrow 0$ and $N \rightarrow \infty$, and product $N p$ aims to some positive constant value $\lambda$ (i.e. $N p \rightarrow \lambda$ ), is Poisson's distribution [13-15]

$$
P(k)=\frac{\lambda^{k}}{k !} \exp (-\lambda), 0 \leq k<\infty .
$$

If sequence of tests with Bernoulli's scheme continues to appear $\mathrm{m}$ "failures", then the number of successes k obeys to negative binomial distribution

$$
P(k)=\frac{\Gamma(m+k)}{\Gamma(m) k !} p^{k}(1-p)^{m}, 0 \leq k<\infty
$$

where $\Gamma(m)$ - gamma function.

Main purpose of this work - to invent sequence probability model of independent tests with three outputs and with it's help receive formulas, analogic to (24), (26) and (27), for defining the probabilities of coming coinciding events.

Let it be produced $N$ of independent tests. Every test can end with three outputs: either event $A$ with the probability $p_{1}$ will come, or event $B$ with the probability $p_{2}$ will come, or event $C$ with the probability $\left(1-p_{1}-p_{2}\right)$ will come. Let's match random discrete value to random output of every test, which takes three values: -1 , if event $A$ happened; 0 , if event $C$ happened and 1 if event $B$ happened. Positive or negative output of every test we'll consider as a "success", and zero output - "failure". In this the probability $P(k)$ of coming events $A, C$ and $B$ in every test can be found by an expression

$$
P(k)=\left\{\begin{array}{cc}
p_{1}, \quad k=-1 ; \\
1-p_{1}-p_{2}, \quad k=0 ; \\
p_{2}, \quad k=1,
\end{array}\right.
$$

where $0<p_{1}<1,0<p_{2}<1, p_{1}+p_{2}<1$

This distribution of probabilities, analogically to Bernoulli's distribution (25), can be called bilateral Bernoulli's distribution. Let's find characteristic function for distribution (28), using ratio [15]

$$
\theta(j \vartheta)=\sum_{k=-1}^{1} \exp (j \vartheta k) P(k)
$$

Substituting in it (28), we'll get

$\theta(j \vartheta)=p_{1} \exp (-j \vartheta)+\left(1-p_{1}-p_{2}\right)+p_{2} \exp (j \vartheta)$
Since ongoing tests are independent, then characteristic function $\theta_{N}(j \vartheta)$ of distribution laws $P(k)$ in $N$ tests will be equal to expression:

$$
\begin{aligned}
& \theta_{N}(j \vartheta)=\theta(j \vartheta)^{N}= \\
& =\left[p_{1} \exp (-j \vartheta)+\left(1-p_{1}-p_{2}\right)+p_{2} \exp (j \vartheta)\right]^{N} .
\end{aligned}
$$

In this probability distribution $P(k)$ in $N$ tests can be found by a formula:

$$
\begin{aligned}
& P(k)=\frac{1}{2 \pi} \int_{-\pi}^{\pi} \theta(j \vartheta)^{N} \exp (-j \vartheta k) d \vartheta \\
& k=-N,-(N-1), \ldots, N
\end{aligned}
$$

Substituting (31) in (32) and integrating, let's find obvious expression for probability distribution $P(k)$ in $N$ tests

$$
P(k)=\left(1-p_{1}-p_{2}\right)^{N} \times\left(\sqrt{\frac{p_{2}}{p_{1}}}\right)^{k} \sum_{i=|k|}^{N} \frac{N !}{(N-i) !} \times B(i, k)\left(\frac{\sqrt{p_{1} p_{2}}}{1-p_{1}-p_{2}}\right)^{i},
$$

Where

$$
B(i, k)=\frac{0,5\left(1+(-1)^{i+|k|}\right)}{\Gamma(0,5(i-k)+1) \Gamma(0,5(i+k)+1)} .
$$

Expression (10) can be simplified for five private cases: 1. If $p_{1}=p_{2}=p<0.5$, then

$$
P(k)=(1-2 p)^{N} \times \sum_{i=k \mid}^{N}\left(\frac{N !}{(N-i) !} \times\left(\frac{p}{1-2 p}\right)^{i} \times \frac{0,5\left[1+(-1)^{i+k \mid}\right]}{\Gamma[0,5(i+k)+1] \Gamma[0,5(i-k)+1]}\right) .
$$

2. If $p_{1}=(1-p)^{2}, p_{2}=p^{2}$, then

$$
P(k)=\frac{(2 N) !}{(N-k) !(N+k) !} \times p^{N+k}(1-p)^{N-k}, k=-N,-(N-1), \ldots, N .
$$

Probability distribution (35), just like distribution (24) is a binomial distribution with not-zero shift parameter.

3. Let's view limiting case for distribution (33), when probability of coming value $C$ is aims to zero, i.e. $\left(p_{1}+p_{2}\right) \rightarrow 1$. In this case every test will end in two outputs: either coming of event $A$ with the probability $(1-p)$, or event $B$ with the probability $p$. To those outputs can be matched discrete random value, which takes two values: -1 , if event $A$ happened and 1 , if event $B$ happened. In this probability distribution (33) in result can be transformed in distribution:

$$
\begin{aligned}
& P(k)=\left(0,5 N !\left[1+(-1)^{N+|k|}\right]\right) \times \\
& (\Gamma[0,5(N+k)+1] \Gamma[0,5(N-k)+1])^{-1} \times\left(\frac{p}{1-p}\right)^{0,5 k}(p(1-p))^{0,5 N} .
\end{aligned}
$$

4. Let's view the second limiting case for distribution (33), when probability of coming event $A$ aims to zero, i.e. 
$p_{1} \rightarrow 0$. In this case every test will end in two outputs: either coming of event $C$ with a probability $(1-p)$, or event $B$ with a probability $p$. To those outputs can be matched random discrete value, which takes two values: 0 , if event $C$ happened and 1 , if event $B$ happened. In this probability distribution (33) as a result of limiting transition transforms in binomial distribution (24). That's why received probability distribution (33) can be called generalized Bernoulli's formula, or bilateral binomial distribution.

5. Let's view the third limiting case for distribution (33), when $p_{1} \rightarrow 0, p_{2} \rightarrow 0, N \rightarrow \infty$, and products $N p_{1}, N p_{2}$ aim to some positive constant values $\lambda_{1}, \lambda_{2}$ (i.e. $N p_{1} \rightarrow \lambda_{1}, \quad N p_{2} \rightarrow \lambda_{2}$ ). In this probability distribution (33) in result of limiting transition transforms in probability distribution

either

$$
P(k)=\exp \left(-\lambda_{1}-\lambda_{2}\right)\left(\sqrt{\frac{\lambda_{2}}{\lambda_{1}}}\right)^{k} \times \sum_{i=|k|}^{\infty} \frac{0,5\left[1+(-1)^{i+|k|}\right]\left(\sqrt{\lambda_{1} \lambda_{2}}\right)^{j}}{\Gamma[0,5(i+k)+1] \Gamma[0,5(i-k)+1]} .
$$

or

$$
P(k)=\exp \left(-\lambda_{1}-\lambda_{2}\right) \times\left(\sqrt{\lambda_{2} / \lambda_{1}}\right)^{k} I_{|k|}\left(2 \sqrt{\lambda_{1} \lambda_{2}}\right),-\infty<k<\infty
$$

where $I_{v}(z)$ - modified Bessel's function.

If parameter $\lambda_{1} \rightarrow 0$, and parameter $\lambda_{2} \rightarrow \lambda$, then distribution (37) or (38) transforms in Poisson's distribution (26). That's why probability distribution (37) or (38) can be called bilateral Poisson's distribution. Characteristic function for it is presented lower

$$
\theta(j \vartheta)=\exp \left[-\left(\lambda_{1}+\lambda_{2}\right)+\lambda_{1} \exp (-j \vartheta)+\lambda_{2} \exp (j \vartheta)\right]
$$

Primary moment of first order and central moments of second order. Third and fourth order for distribution (33) can be found from the expressions

$$
\begin{aligned}
& m_{1}=N\left(p_{2}-p_{1}\right) ; \\
& M_{2}=N\left[p_{2}+p_{1}-\left(p_{2}-p_{1}\right)^{2}\right] ; \\
& M_{3}=\left(p_{2}-p_{1}\right) \times\left[N-N\left(p_{2}-p_{1}\right)^{2}-3 M_{2}\right], \\
& M_{4}=M_{2}\left[1+6\left(p_{2}-p_{1}\right)^{2}\right]+3(1-1 / N) M_{2}^{2}+ \\
& +3 N\left(p_{2}-p_{1}\right)^{2}\left[\left(p_{2}-p_{1}\right)^{2}-1\right] .
\end{aligned}
$$

Instead of central moments of the third and the fourth orders usually use asymmetry coefficient $K_{a}$ and excess coefficient $K_{e}$, which can be found from ratios
$K_{a}=\frac{M_{3}}{M_{2}^{1,5}}$

$K_{e}=\frac{M_{4}}{M_{2}^{2}}-3$.

Expressions (40)-(42) for moments are significantly simplified for private distribution cases (33). So, for distribution (34), for mentioned moments the fair expression is:

$$
\begin{aligned}
& m_{1}=0 ; \\
& M_{2}=2 N p ; \\
& M_{3}=0 ; \\
& M_{4}=M_{2}+3(1-1 / N) M_{2}^{2} .
\end{aligned}
$$

In this

$$
K_{a}=0 ; K_{e}=\frac{0,5-3 p}{p N}
$$

For distribution (45) fair expressions are:

$$
\begin{aligned}
& m_{1}=N(2 p-1) ; \\
& M_{2}=2 N p(1-p) ; \\
& M_{3}=2 N p(1-p)(1-2 p) ; \\
& M_{4}=2 N p(1-p) \times[1+6 p(1-p)(N-1)] . \\
& K_{a}=\frac{1-2 p}{\sqrt{2 N p(1-p)}}, \\
& K_{e}=\frac{1-6 p(1-p)}{2 N p(1-p)} .
\end{aligned}
$$

For distribution (36) fair expressions are:

$$
\begin{aligned}
& m_{1}=N(2 p-1) ; \\
& M_{2}=4 N p(1-p) ; \\
& M_{3}=8 N p(1-p)(1-2 p) ; \\
& M_{4}=3 M_{2}^{2}+4 M_{2}[1+6 p(1-p)], \\
& K_{a}=\frac{1-2 p}{\sqrt{N p(1-p)}} \\
& K_{e}=\frac{1+6 p(1-p)}{N p(1-p)} .
\end{aligned}
$$
have

From this follows, that for expression (37) or (38) we 


$$
\begin{aligned}
& m_{1}=\lambda_{2}-\lambda_{1}, \\
& M_{2}=\lambda_{1}+\lambda_{2}, \\
& M_{3}=\lambda_{2}-\lambda, \\
& M_{4}=\lambda_{1}+\lambda_{2}+3 M_{2}^{2}, \\
& K_{a}=\frac{\lambda_{2}-\lambda_{1}}{\left(\lambda_{1}+\lambda_{2}\right)^{1,5}}, \\
& K_{e}=\frac{1}{\lambda_{1}+\lambda_{2}} .
\end{aligned}
$$

Probability $P_{B}(k)$ of fact, that event $B$ in $N$ tests will come $\mathrm{k}$ times can be found from formula (33), or from it's private cases (34), (35), (36), (37) or (38). In this we suppose, that $P_{B}(k)=P(k), k=1,2, \ldots, N$.

Probability $P_{A}(k)$ of fact, that event $A$ in $N$ tests will come $k$ times can be also found from formula (33), or it's private cases (34), (35), (38), (37) or (38). In this we suppose, that $P_{A}(k)=P(k), k=-1,-2, \ldots,-N$.

Probability $P_{C}$ of coming event $C$ in $N$ tests can be found using formula (33), or it's private cases (34), (35), (36), (37) or (38). In that we suppose, that $P_{C}=P(0)$. Probability $P_{C}$ matches to probability of fact, that in $N$ cases events $A$ and $B$ won't come.

Let's view the example. Two symmetric coins are being thrown for ten rimes. In every throw three outputs are possible: two "eagles" with probability 0,25 ; two "tails of coin" with probability 0,25 and "eagle and tail of coin" with probability 0,5 . It's necessary to find: 1$)$ probability $P_{e e}$ of fact, that precisely five times two "eagles" drop; 2) probability $P_{t t}$ of fact, that precisely three times two "tails of coin" drop; 3) probability $P_{e t}$ of fact, that precisely five times two "eagles" and three "tales of coin" drop.

Solution: In the match with example's condition we have

$$
\begin{aligned}
& p_{1}=p_{2}=p=0.25, \\
& N=10 ; P_{e e}=P_{A}(-5), \\
& P_{t t}=P_{B}(3) ; P_{e t}=P_{A}(-5) P_{B}(3) .
\end{aligned}
$$

I.e. $p_{1}=p_{2}$, then we use expression (9) as a counting formula. With it's help we find, that either

$$
\begin{aligned}
& P_{e e} \approx 0,015 ; \\
& P_{t t} \approx 0,074 ; \\
& P_{e t} \approx 1,093 \cdot 10^{-3} ;
\end{aligned}
$$

or

$$
\begin{aligned}
& P(k)=\left(1-p_{1}-p_{2}\right)^{m} \times \\
& \left(\sqrt{\frac{p_{2}}{p_{1}}}\right)^{k} \times\left(\sqrt{p_{1} p_{2}}\right)^{|k|} \frac{\Gamma(m+|k|)}{\Gamma(m)} F(k),-\infty<k<\infty,
\end{aligned}
$$

where $F(k)={ }_{2} F_{1}\left(0,5(m+|k|), 0.5(m+|k|+1), 1+|k|, 4 p_{1} p_{2}\right)$

- Hypergeometric Gaussian function.

Characteristic function of distribution (54) or (55 has the view

$\theta(j \vartheta)=\left[\left(1-p_{1}-p_{2}\right) \times\left(1-p_{1} \exp (-j \vartheta)-p_{2} \exp (j \vartheta)\right)^{-1}\right]^{m} .(56)$

Primary moment of the first order and central moments of the second, the third and the fourth orders for expressions (54) or (55) are defined by expressions

$$
\begin{aligned}
& m_{1}=\frac{m\left(p_{2}-p_{1}\right)}{1-p_{1}-p_{2}} ; \\
& M_{2}=\frac{m\left(p_{2}+p_{1}-4 p_{1} p_{2}\right)}{\left(1-p_{1}-p_{2}\right)^{2}} ; \\
& M_{3}=\frac{m\left(p_{2}-p_{1}\right)}{\left(1-p_{1}-p_{2}\right)^{3}} \times\left(1+p_{2}+p_{1}-8 p_{1} p_{2}\right) ; \\
& M_{4}=m\left[\frac{6\left(p_{2}-p_{1}\right)^{4}}{\left(1-p_{1}-p_{2}\right)^{4}}+\left(\frac{4\left(p_{2}-p_{1}\right)^{2}}{\left(1-p_{1}-p_{2}\right)^{3}}+\frac{\left(p_{1}+p_{2}\right)}{\left(1-p_{1}-p_{2}\right)^{2}}\right)\right. \\
& \left.\times\left(2 p_{1}+2 p_{2}+1\right)\right]+3 M_{2}^{2} .
\end{aligned}
$$

Let's view limiting case for distribution (31) or (32), when probability $p_{1} \rightarrow 0$, and probability $p_{2}=p$. In this probability distribution (31) or (32) as a result of limiting transaction transforms in negative binomial distribution (4). That's why received probability distribution (31) or (32) can be called bilateral negative binomial distribution.

Choosing from bilateral binomial, Poisson's and negative binomial distributions we can use following properties of those distributions: Binomial $-K_{e} M_{2}<1$, Poisson's $-K_{e} M_{2}=1$, Negative binomial $-K_{e} M_{2}>1$.

So, there was developed probability model for sequence of independent tests with three outputs, were received expressions for it's general number characteristics, and also for calculating the probabilities of coming matched events precisely k times. Was shown, that limiting cases of received bilateral distributions are binomial, negative binomial and Poisson's distributions.

\section{Conclusion}

In this way, the following results are obtained.

- Generalized expressions for one-way and two-way continuous distribution laws with maximum entropy depending on the number of existing power, exponential or logarithmic moments. With their help, one can more reasonably choose the a priori distribution under the conditions of a priori uncertainty in the analysis of the risks of information systems. From the analysis of expression (23) and its particular cases (2), (11), (14), (17), (20) at the appropriate values $q(x)$ it follows that in the general case the entropy depends also on the type of moments used to 
determine the numerical characteristics of the distribution law.

- Probabilistic model for a sequence of independent trials with three outcomes, which acquire special significance in the formation of information security assessments of information systems. Expressions for its basic numerical characteristics are obtained. It is shown that the limiting cases of the obtained two-way distributions are the binomial, negative binomial and Poisson distributions.

This is an Open Access article distributed under the terms of the Creative Commons Attribution License

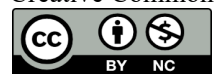

\section{References}

[1] Burkov V., Novikov D., Shchepkin A. Control Mechanisms for Ecological-Economic Systems. - Berlin: Springer, 2015. - 174 p.

[2] Kuznecov N.A., Kul'ba V.V., Mikrin E.A., Kovalevskij S.S., Pavlov B.V., Kosjachenko S.A., Malineckij G.G., Arhipova N.I., Kul'ba A.V., Volkov A.E., Shelkov A.B., Vlasov S.A., Kononov D.A., Shubin A.N., Chernov I.V., Gladkov Ju.M. Informacionnaja bezopasnost' sistem organizacionnogo upravlenija. Teoreticheskie osnovy [Information security of organizational management systems. Theoretical basis]. V 2-h tomah. Tom 1. M.:Nauka, 2006. $-495 \mathrm{p}$.

[3] Kuznecov N.A., Kul'ba V.V., Mikrin E.A., Kovalevskij S.S., Pavlov B.V., Kosjachenko S.A., Malineckij G.G., Arhipova N.I., Kul'ba A.V., Volkov A.E., Shelkov A.B., Vlasov S.A., Kononov D.A., Shubin A.N., Chernov I.V., Gladkov Ju.M. Informacionnaja bezopasnost' sistem organizacionnogo upravlenija. Teoreticheskie osnovy [Information security of organizational management systems. Theoretical basis]. V 2-h tomah. Tom 2. M.: Nauka, 2006. $-437 \mathrm{p}$.

[4] Gromov Yu.Yu., Ivanovskiy M.A., Ivanova O.G., Yakovlev A.V. Analiz i sintez struktur informatsionnykh tselenapravlennykh system [Analysis and synthesis of structures of informationoriented systems] - Saarbryuken (Germaniya): LAP LAMBERT Academic Publishing. - 2015. - 164 p.

[5] Shul'c V.L., Kul'ba V.V., Shelkov A.B., Chernov I.V., Somov D.S. Upravlenie tehnogennoj bezopasnost'ju na osnove scenarnogo i indikatornogo podhodov [Managing technogenic safety based on scenario and indicator approaches]. M.: IPU RAN, 2013.-116 p.

[6] Shul'c V.L., Kul'ba V.V., Shelkov A.B., Chernov I.V. Metody scenarnogo analiza ugroz jeffektivnomu funkcionirovaniju system organizacionnogo upravlenija [Methods of scenario analysis of threats to the effective functioning of organizational management systems] // Trendy i upravlenie. - 2013. - No 1. - P. 6-30. DOI: 10.7256/2307-9118.2013.01.2.

[7] Gromov Yu.Yu., Ivanovskiy M.A., Didrikh V.E., Ivanova O.G., Martem'yanov Yu.F. Metody analiza informatsionnykh system [Methods of analysis of information systems]. Tambov; M.; SPb.;
Baku; Vena; Gamburg: Izd-vo MINTS «Nobelistika», 2012. 220 .

[8]Gromov Yu.Yu., Karpov I.G. Dal"nejshee razvitie sushhestvujushhih predstavlenij ob osnovnyh formah zakonov raspredelenij i chislovyh harakteristik sluchajnyh velichin dlja reshenija zadach informacionnoj bezopasnosti [Further development of existing representations on main forms of laws of distribution and numerical characteristics of random values for solving the problems of information security] // Informacija i bezopasnost'. - 2010. - T. 13. No 3. - P. 459-462.

[9] Teoriya informatsionnykh protsessov i system [Theory of information processes and systems] / Yu. Yu. Gromov, V. E. Didrikh, O. G. Ivanova, V. G. Odnol'ko. - Tambov : Izd-vo FGBOU VPO «TGTU», 2014. - 172 p.

[10] Gromov Yu. Yu. Generalized probabilistic description of homogeneous flows of events for solving informational security problems / Y.Y. Gromov, I.G. Karpov, Y.V. Minin, O.G. Ivanova // Journal of Theoretical and Applied Information Technology. 2016. - T. 87. - № 2. - P. 250-254.

[11] Gnedenko B.V. Kurs teorii veroyatnostey [Course of probability theory]. - M.: Nauka, 1988. - 448 p.

[12] Levin B.R. Teoreticheskiye osnovy statisticheskoy radiotekhniki [Theoretical bases of statistical radio engineering]. - M.: Radio i svyaz', 1989.

[13] Veroyatnost' i matematicheskaya statistika: entsiklopediya [Probability and mathematical statistics: encyclopedia] / Gl. red. YU.V. Prokhorov. - M.: Bol'shaya Rossiyskaya entsiklopediya, 1999. -910 p.

[14] Odnomernyye diskretnyye raspredeleniya [One-dimensional discrete distributions] / N.L. Dzhonson, S. Kots, A. Kemp. - M.: BINOM. Laboratoriya znaniy, 2010. - 559p.

[15] Vadzinskiy R.N. Spravochnik po veroyatnostnym raspredeleniyam [Handbook of probability distributions]. - SPb.: Nauka, 2001. 296 p. 